\title{
Dificultades de estrategia en el resumen de textos $(*)(* *)$
}

\section{Peter N. Winograd}

\author{
Universidad de Kentucky
}

Traducción: Silvia Sánchez-Herrero

En los últimos años, los investigadores han empezado a saber algo sobre los problemas de orden superior en la comprensión, que contribuyen posiblemente a la frustración que experimentan algunos niños en la lectura. La expresión «de orden superior» se refiere a aquellos problemas que no se deben a unas destrezas inadecuadas en la decodificación ni a problemas en el acceso lexical (Golinkoff, 1975-76). Las investigaciones recientes han enfatizado algunos de los posibles origenes de tales dificultades, entre los que se incluyen los relacionados con experiencias lingüísticas diferenciales, con una falta de conocimiento previo o con una ausencia de destrezas de estrategia (Collins y Havilland, 1979). Es precisamente esta tercera posibilidad, la carencia de unas destrezas de estrategia, el punto central de este artículo.

El propósito de esta investigación era examinar sistemáticamente las diferencias de estrategia entre los lectores deficientes y los buenos lectores cuando resumían lo que habían leido. Habia tres aspectos interesantes en el uso de estrategias: (a) la conciencia de las demandas de la tarea implicadas al hacer resúmenes, (b) la aptitud para identificar los elementos importantes del texto y (c) la aptitud para transformar y resumir el significado de un texto a lo esencial. Estos aspectos nos proporcionaron la base para establecer tres hipótesis experimentales acerca de por qué los lectores deficientes podrían tener dificultades a la hora de hacer resúmenes adecuados:
1. Los lectores deficientes no son conscientes de que el propósito del resumen sea expresar las ideas importantes de una manera concisa:

2. Los lectores deficientes fracasan al identificar la información que, ségún el criterio de los adultos, debería incluirse en un resumen.

3. Los lectores deficientes' no alcanzan a utilizar o utilizan ineficazmente, las transformaciones que usan los buenos lectores.

En cada una de estas tres hipótesis había tres objetivos: (a) identificar y caracterizar los posibles déficit de estrategia de los lectores deficientes, (b) examinar la influencia que tales déficit tendrian en la aptitud para realizar la tarea de resumir y (c) examinar la influencia que esos déficit tendrian en la aptitud para comprender lo que se había leído, para determinar si el uso de la estrategia va más allá de la tarea de resumir.

En este estudio la aptitud para resumir se definió como la aptitud para «expresar los puntos principales de una forma concisa" (Webster's Third New International Dictionary, 1964). Los puntos principales se definieron operativamente como aquellas ideas identificadas especificamente como importantes según un criterio de clasificación o selección de lectores adultos fluidos, o como aquellas ideas incluidas con más frecuencia en los resúmenes de esos mismos lectores. Aunque este enfoque aclaraba poco la importacia teórica, nos proporcionaba

* Este artículo está basado en un informe que quedó entre los diez finalistas del IRA's Outstanding Dissertation Award en su convocatoria de 1980-81. En la convención anual del IRA de 1982 celebrada en Chicago, se presentó una ponencia basada en ese informe.

** "Strategic difficulties in summarizing texts" Reading Research Quarterly, 1984, XIX, 4, 404-425. Reproducido con autorización (C) de traducción Infancia y Aprendizaje, 1985. 
una vía para examinar las diferencias debidas a la edad y a la aptitud.

Se eligió el resumen como tarea experimental por varias razones. Primero, porque la aptitud para obtener los puntos principales o lo esencial de lo que uno ha leído, es de gran importancia y muchos estudiantes tienen dificultades en esta tarea (Kennedy, 1971). Segundo, porque había suficiente investigación que se centraba en las estrategias implicadas en el resumen, proporcionándonos un marco de referencia conceptual para analizar la tarea (Brown y Day, 1980; Kintsch y van Dijk, 1978). Tercero, porque aunque la relación no está muy clara, algunas de las estrategias usadas al resumir, pueden usarse también en la comprensión y entonces, los resultados derivados del estudio de los resúmenes nos proporcionarian información acerca de los procesos de la comprensión en general (Kintsch y van Dijk, 1978; Johnston, 1978, y Johnston, 1981).

El modelo propuesto por Kintsch y van Dijk (1978) fue elegido como marco teórico para este estudio, puesto que intenta demostrar cómo las oraciones del texto se transforman y se condensan en lo esencial. Los componentes esenciales de este modelo son: el esquema del lector, la microestructura y macroestructura del texto y un conjunto de macro-reglas para hacer resúmenes. De acuerdo con este modelo; los lectores avanzan a través del texto organizando y reduciendo la microestructura en una macroestructura, gracias a la aplicación de una serie de transformaciones conocidas como macro-reglas.

Estas macro-reglas no se aplican de forma fortuita, sino que más bien estản restringidas por los propósitos del lector, que determinan qué elementos del texto son relevantes y cuáles no. Los elementos son relevantes según dos criterios (van Dijk, 1979). El primero es la importancia del texto, que sé define en términos de lo que el autor considera importante y a menudo se indica mediante unas señales en la estructura del mismo. El segundo criterio es la importancia contextual que proviene de los intereses personales del lector o de sus conocimientos. Los lectores fluidos pueden usar tanto el criterio del propio texto como el contextual, de manera que se asigna importancia a los elementos que son personalmente relevantes y a los elementos que el autor del texto tiene la intención de que sean relevantes.

La investigación que presentamos aquí trata de tres aspectos. interrelacionados del modelo de Kintsch y van Dijk: (a) la adecuación de los objetivos del lector, (b) la identificación de aquellos elementos más importantes del texto y (c) el uso de las macro-reglas. Se seleccionaron estos aspectos puesto que la literatura sobre el tema sugiere que estas tres dimensiones pueden suponer dificultad a los lectores deficientes.

Primero, los estudios indican que algunas dificultades en la lectura del niño, pueden estar relacionadas con la confusión sobre los fines adecuados de diversas tareas de la lectura (por ejemplo, Baker y Brown, 1980; Downing, 1979). La mayor parte de la investigación que lo sustenta, se ha centrado en la adquisición de las destrezas en la lectura temprana (por ej., Clay, 1969; Denny y Weintraub, 1963; Ehri, 1979; Johns, 1980; Redi, 1966). Sin embargo, los trabajos de varios investigadores (Canney y Winograd, 1979; Meyers y Paris, 1978) han indicado que incluso los estudiantes mayores pueden confundirse también en las demandas de la tarea de la lectura. Asi, algunas dificultades de los-lectores deficientes a la hora de resumir, pueden deberse a las confusiones sobre las demandas de la tarea.

Segundo -los estudios Kan demostrado también, que los buenos lectores y los deficientes se diferencian en su sensibilidad hacia lo importante (Dunn, Mathews y Bieger, 1979; Eamon, 1978; Meyer, Brandt y Bluth, 1980; Smiley, Cakley, Worthen, Campione y Brown, 1977). Sin embargo, las preguntas sobre la naturaleza y los efectos de estas diferencias permanecen aún sin contestar. ¿Difieren los lectores deficientes de los buenos lectores de una forma sistemática en lo que consideran importante en los textos? Parafraseando a Brown y Smiley (1977), los juicios de importancia podrian diferir porque los lectores deficientes tienen una concepción distinta de lo que es importante o porque les falta sensibilidad hacia lo importante. La relación entre la sensibilidad hacia lo importante y las dificultades en la comprensión lec- 
tora, no está todavía muy clara. El tema es, si la sensibilidad hacia lo importante justifica o no las diferencias individuales en la comprensión, más que los problemas de decodificación en un nivel inferior (Berger y Perfetti, 1977; Perfetti y Lesgold, 1977; Pichert, 1979).

Tercero, algunos estudios recientes (Brwon y Day, 1980; Day, 1980) han demostrado que los buenos lectores y los deficientes se diferencian en su aptitud para aplicar las diversas transformaciones (a las que nos referiremos como reglas) usadas en un resumen. También aquí existen preguntas acerca de la naturaleza y de los efectos de estas diferencias. $¿$ Cuál de estas transformaciones es la más difícil? ¿Cómo se relacionan directamente las diferencias en la aptitud para usar transformaciones y aquello que se comprende y se resume?

En conclusión, aunque la literatura sugiere que los lectores buenos y los deficientes difieren en las dimensiones de la conciencia de la tarea, de la sensibilidad hacia lo importante y del uso de las reglas para resumir, existen aún muchas preguntas sin responder. Por otra parte, ningún estudio anterior ha considerado las tres dimensiones usando la misma población. Así, la tarea de resumir y el marco de referencia conceptual de Kintsch y van Dijk proporcionan una oportunidad única para estudiar las dificultades de estrategia en los lectores deficientes.

\section{METODO}

\section{Sujetos}

La muestra inicial estuvo formada por 80 estudiantes del grado 8 y por 40 adultos. Se consideraron lectores deficientes a aquellos niños que puntuaron por debajo del percentil $50^{\circ}$ en el subtest de comprensión lectora del Stanford Achievement Test. La puntuación media de estos sujetos fue 26,94 (DT $=9,84)$. Se definieron como buenos lectores a aquellos niños que puntuaron por encima del percentil 59 en el mismo test. La media de estos sujetos fue 66,28 (DT $=9,59$ ). Todos los adultos estaban relacionados con la Universidad de Illinois, bien como estudiantes, como licenciados o como licenciados en estudios de doctorado. La ausencia y los datos incompletos redujeron los sujetos a 36 lectores deficientes, 39 buenos lectores y 37 adultos.

\section{Material}

El primer grupo de materiales consistió en una serie de preguntas de elección múltiple y de respuestas cortas diseñada para determinar el conocimiento de los sujetos sobre la tarea de resumir. Antes que nada, se formularon las siguientes preguntas:

1. ¿Con qué frecuencia te piden en la escuela que resumas lo que has leído?

2 . ¿Te ha enseñado alguien cómo resumir lo que has leido?

3. Si tu respuesta es «si» ¿qué te han enseñado a hacer?

4. ¿Qué quiere decir resumir un texto?

Después de haber realizado todas las tareas experimentales se formularon las siguientes preguntas. La n. ${ }^{\circ} 4$ se repitió para evaluar los posibles efectos del experimento.

1. ¿Qué quiere decir resumir un texto?

2. Enumera al menos tres maneras de decidir qué ideas de un artículo deberían incluirse en un resumen.

3. Enumera al menos tres cosas en las que tus resúmenes sean diferentes de los textos originales.

El siguiente grupo de material experimental consistió en 8 pasajes adaptados de libros sobre comercio, estudios sociales elementales, ciencias y.textos de lectura (Education Development Center, 1970; Ginn Basic Reader, 1967; Holt Databank System, 1976a, 1976b; Noble y Noble, 1974; Wright, 1971). Todos los trozos escogidos tenían aproximadamente el mismo número de palabras $(\overline{\mathrm{X}}=$ $344 ; \mathrm{DT}=18,35$ ) y oscilaban entre el grado tercero superior y el grado sexto inferior en dificultad (Fry, 1977).

Los ocho textos fueron la base de otros materiales experimentales. Primero un test de elección múltiple de cinco preguntas para cada texto, que se diseñó 
para medir la comprensión de información específica de ese pasaje. Las alternativas y las respuestas correctas se tomaron de información explícita expresada en el texto.

Luego se desarrolló una lista que contenia 100 palabras elegidas al azar de los textos (sin incluir los nombres propios). Esta lista se diseñó para obtener un coeficiente de decodificación y unas medidas de precisión para cada uno de los sujetos del grado ocho.

El último conjunto de materiales se creó tomando cada texto y haciendo una lista de cada una de sus frases. Estas se numeraron consecutivamente y se añadió una escala de cinco puntos para que los sujetos pudieran evaluar la importancia que tenía esa frase en el texto.

\section{Procedimiento}

Cada uno de los estudiantes del grado ocho fue asignado al azar a uno de los cuatro grupos formados de igual tamaño, con la única restricción de que tendria que haber igual número de lectores buenos y deficientes en cada grupo. $\mathrm{El}$ orden de los textos fue contrabalanceado en cada grupo.

Los datos iniciales de los estudiantes del grado ocho se recogieron en dos momentos en un periodo de tres semanas. Cada niño participaba en ocho sesiones de 40 minutos de duración. En una primera etapa, se midió a los niños individualmente para obtener las medidas de la velocidad y de la precisión en la decodificación. Durante este tiempo, también se les aplicó el primer grupo de preguntas de forma escrita y el IPAT Culture Fair (Cattell y Cattell, 1960 ).

En una segunda etapa, los estudiantes del grado ocho completaron el resto de las tareas experimentales. El orden de aplicación de estas tareas fue el mismo para todos los sujetos. Primero, los niños leían un texto y se registraba el tiempo de lectura. Luego contestaban a las cinco preguntas de elección múltiple sin tener acceso al texto. Cuando habian terminado de contestar las preguntas, realizarón un resumen de unas 60 palabras teniendo el artículo delante. Después de una breve tarea interpolada, los sujetos estimaron la importacia relativa de cada frase en el total del texto. En la última tarea, los sujetos volvían a las frases numeradas y seleccionaban las cinco más importantes.

Después del último paso, se dio a los niños un momento de descanso antes de proseguir con el siguiente texto y repetir el proceso. Cada niño trabajó con un total de 6 de los 8 pasajes y cada texto lo leyeron el mismo número de sujetos. Además, la tarea del resumen y las tareas de selección y clasificación se contrabalancearon de forma que el niño resumiera y luego identificara la importancia de los tres primeros textos y se invirtió el orden en los tres últimos. Cuando se había realizado el resumen final, los niños contestaron a las preguntas que faltaban de la entrevista sobre la conciencia de la tarea. Todas las respuestas se daban de forma escrita.

Seis meses después, se recog̈ieron los datos finales de un subgrupo de lectores buenos $(n=12)$ y de lectores deficientes $(n=12)$. A estos niños se les pidió que seleccionaran la información importante de dos pasajes que habían visto anteriormente. El propósito de esta tarea final fue obtener una medida de la fiabilidad a largo plazo de los índices de importancia dados por los niños.

Los datos de los adultos se recogieron en una única sesión de dos horas. Cada adulto trabajó con los ocho textos y todas las tareas fueron las mismas, excepto que no se les aplicó el IPAT Culture Fair Test, ni las medidas de decodificación ni la última tarea para indicar lo importante.

\section{Puntuación de los resúmenes}

Los resumenes se puntuaron usando un sistema (Winograd, 1982) que hacía posible identificar qué ideas del pasaje original se incluían en el resumen, así como registrar qué transformaciones se habían empleado en esas ideas. Se identificaron cuatro grandes categorias de transformaciones: repeticiones, combinaciones, combinaciones enlazadas e invenciones.

Las repeticiones se refieren a los ejemplos en los que los sujetos reproducian frases del texto original, bien copiadas literalmente, bien mediante una paráfrasis. 
Las combinaciones son las transformaciones en donde los sujetos combinaban dos o más frases del texto original en una sola en el resumen. Por ejemplo, la siguiente combinación está extraída del resumen de un adulto (el texto original está en el apéndice).

La nutria de río es muy parecida a la nutria marina, pero es más pequeña, come peces, ranas y reptiles y va por tierra.

Las combinaciones enlazadas se refieren a los ejemplos en los que los elementos de diversas frases del pasaje original se han incluido en el resumen, pero de un modo menos organizado que las combinaciones. Se introdujo esta categoria porque era necesario distinguir entre las combinaciones bien hechas y aquellas producidas por los sujetos que incluían palabras en forma lineal menos organizada a lo largo de las frases. Un ejemplo del resumen de un lector deficiente ayudará a clarificar la distinción entre las combinaciones normales y las combinaciones enlazadas.

Las nutrias marinas, cuerpos estrechos, piernas cortas, los rabos son gordos y una forma plana les ayuda a nadar en la profundidad, comen del mar, comen peces, ranas, reptiles en el agua la nutria de río más pequeña y no tan pesada doblan las patas de delante y empujan, juegan, bajan deslizándose por la orilla del río.

Las invenciones se refieren a los ejemplos en los que el sujeto produce frases que transmiten el significado de un párrafo, de varios o incluso de todo el texto. Cuando estas frases se relacionaban con el pasaje, era muy difícil vincularlas a algún elemento específico de la estructura superficial de las frases originales. Estas invenciones eran a menudo frases que cubrían la información de un modo general. Aquí tenemos un ejemplo de uno de los adultos.

Las nutrias de mar son diferentes de las de rio en la apariencia y en los hábitos.

Como un control de la fiabilidad del sistema de puntuación, se examinó un segundo índice del 20 por 100 de los resúmenes realizados por los tres grupos.
Los coeficientes de fiabilidad para identificar qué ideas de los pasajes originales estaban incluidas en los resúmenes fueron: 0,96 para los lectores deficientes, 0,94 para los buenos lectores y 0,89 para los adultos. La fiabilidad interindices sobre el acuerdo de las transformaciones usadas, fue ligeramente inferior: 0,93 para los lectores deficientes, 0,91 para los buenos lectores y 0,84 para los adultos.

Este sistema de puntuación difiere de otros usados normalmente en varios aspectos (Brown y Day, 1980; van Dijk, 1977). La diferencia más importante es que en este sistema, se categorizaron las transformaciones considerando simplemente el modo en que se modificaba la información original para hacer el resumen y, una vez llegados a este punto, no se tomaban decisiones sobre la importancia relativa de la información modificada, tal y como hicieron Brown y Day (1980) y van Dijk (1977). Por ejemplo, Brown y Day usaron el término invención para referirse a la producción de una frase temática referida a un párrafo que carecía de una apropiada. En este sistema, sin embargo, no se determinó si la transformación culminaba o no en una nueva frase temática. La nueva frase se puntuaba bien como una combinación bien como una invención, dependiendo de si los elementos se referían o no a elementos especificos del texto original.

Aunque el sistema de puntuación usado en este estudio se inspira fuertemente en el trabajo de van Dijk (1977) y en el de Brown y Day (1980), resulta más sencillo de aplicar a los resúmenes producidos a partir de los textos que se dan en situaciones habituales, puesto que quien los califica sólo ha de decidir qué transformaciones han tenido lugar. Posteriormente, en un análisis, por separado pueden tomarse decisiones sobre la importancia relativa de la información que ha sido modificada.

\section{RESULTADOS}

Los análisis se realizaron sobre tres conjuntos de datos. El primer grupo, las respuestas de los sujetos a las preguntas de la entrevista, se usó para determinar la conciencia de las demandas de la tarea 
de resumir. El segundo conjunto, los indices de importancia de los temas y de las selecciones, se usó para examinar la sensibilidad del sujeto hacia lo importante. El tercer grupo, los resúmenes, se utilizó para examinar qué transformaciones se habían aplicado. Los principales hallazgos se resumen más abajo.

En todos los análisis apropiados se utilizaron los coeficientes de correlación transformados Fisher Z. Sin embargo, presentaremos los coeficientes de correlación sin transformar para facilitar la comunicación y la interpretación. Las medidas post hoc fueron las de Scheffé con un nivel de significación del 0,50 . Se realizaron comprobaciones sobre los efectos principales simples, respecto a todas las interacciones significativas, empleando procedimiento recomendado por Kirk (1968). Los niveles de significación se determinaron concretamente dividiendo el indice alfa general por la medida del efecto principal, equitativamente entre el conjunto de las medidas de los efectos principales simples.

\section{Conciencia de la tarea}

A todos los sujetos se les formuló una serie de preguntas para determinar el conocimiento que tenian sobre la tarea de resumir. Las dos primeras preguntas fueron de elección múltiple y se analizaron fácilmente. En todas las preguntas abiertas, las respuestas se analizaron identificando las de varios sujetos y ordenándolas según la proporción de sujetos que las hubiesen producido. Estas respuestas ordenadas se analizaron luego usando el coeficiente de correlación de Goodman y Kruskal (Blalock, 1979).

Los detalles relativos a las respuestas de los sujetos a las preguntas, pueden encontrarse en Winograd (1982). En resumen, el 88 por 100 de los lectores deficientes, el 92 por 100 de los buenos lectores y el 89 por 100 de los adultos afirmaron que al menos alguna vez se les pidió que hicieran resúmenes en la escuela. Además, el 74 por 100 de los lectores deficientes, el 72 por 100 de los buenos lectores y el 54 por 100 de los adultos confesaron que les habían enseñado cómo resumir.

Recordemos que la pregunta «QQué quiere decir resumir un texto?» se for- muló dos veces para determinar los posibles efectos del experimento. Los ligeros cambios experimentados en la reducción proporcional de error entre la primera y la segunda vez en que se hizo la pregunta, indicaron un patrón de respuesta relativamente estable.

El análisis más pertinente se centró en la importancia de las ideas. Teóricamente, la característica más sobresaliente de un resumen, es que contiene las ideas más importantes del texto original. Por lo tanto, si un sujeto mencionaba explícitamente los puntos importantes o las ideas principales, al menos una vez en una respuesta a cualquiera de las preguntas, se le asignaba la puntuación de 1 , que indicaba la conciencia de este aspecto del resumen. Si el sujeto no hacía referencia explícita a la importancia pero daba alguna respuesta imprecisa acerca de lo que trataba el artículo, se le asignaba una puntuación de 2 . Se hizo esta distinción para ver si una referencia a la importancia era un índice de una conciencia metacognitiva mejor desarrollada respecto a las demandas de la tarea. Si un sujeto no se refería nunca a la importancia, ni a aquello de lo que trataba el texto en sus respuestas a las preguntas, se le asignaba una puntuación de 3. Estas puntuaciones están basadas en una escala nominal y se usaron posteriormente en una análisis de regresión múltiple para examinar los posibles efectos de la conciencia-de la tarea en varias medidas dependientes.

Los resultados indicaron que el 69 por 100 de los lectores deficientes y el 69 por 100 de los buenos lectores obtuvieron una puntuación de 1 . Al 17 por 100 de los lectores deficientes y al 28 por 100 de los buenos lectores se les asignó una puntuación de nivel de respuesta 2 . El resto de los niños, el 14 por 100 de los lectores deficientes y el 3 por 100 de los buenos lectores, recibieron una puntuación de 3. Puesto que muchos de los estudiantes hicieron referencias explícitas al hecho de que un aspecto del resumen fuera incluir las ideas importantes $o$ al menos establecieron que un resumen indicaba aquello acerca de lo que trataba un artículo, puede concluirse que la confusión sobre el propósito de la tarea no era un problema importante para estos estudiantes del grado 8. Sin embargo, 
dado el escaso refinamiento de esta medida escrita realizada individualmente, no podemos excluir la posibilidad de que haya alguna diferencia sutil entre los lectores deficientes y los buenos lectores.

\section{Sensibilidad hacia lo importante}

El siguiente conjunto de análisis examinó la aptitud de los sujetos para identificar los elementos importantes de los pasajes. Se calcularon las relaciones entre las diversas tareas experimentales en un nivel intraindividual, en un nivel interindividual, y en un nivel de grupo total. Puesto que los resultados en todos los niveles fueron parecidos sólo usaremos, para ilustrar la discusión, las correlaciones más pertinentes. Una comparación preliminar entre la distribución de los índices de los lectores deficientes y la distribución de los índices de los buenos lectores, indicaba que ambos grupos estaban usando toda la gama de cinco puntos y que las formas de estas dos distribuciones se aproximaban a la curva normal. Por otra parte, la variación intrasujeto en los lectores deficientes fue más o menos igual a la de los buenos lectores. Por ejemplo, después de un intervalo de seis meses el coeficiente de fiabilidad test-retest para los lectores deficientes se igualó al de los buenos lectores. La correlación media entre la primera y la segunda estimación fue de 0,20 para ambos grupos. Otro dato proviene de la correlación biserial puntual intraindividual, entre las puntuaciones de la importancia de la frase y las frases seleccionadas como las más importantes. Las correlaciones medias biseriales puntuales fueron 0,524 para los lectores deficientes; 0,485 para los buenos lectores y 0,567 para los adultos. Por tanto, dado que los lectores deficientes han hecho algunos juicios sobre la importancia relativa de las ideas de los textos y puesto que estos juicios eran de alguna forma consistentes, pudieron estimarse los temas relacionados con la sensibilidad de los lectores deficientes hacia lo importante.

El primer gran hallazgo de este conjunto de análisis no fue inesperado. Los buenos lectores hacen mejores juicios de la importancia que los lectores deficientes, cuando lo importante se definió en los términos del adulto. Consideremos las correlaciones halladas en el grupo to- tal entre los índices de importancia media de cada grupo. La correlación entre los lectores deficientes y los adultos fue de 0,459; entre los buenos lectores y los adultos fue de 0,708 .

El segundo hallazgo principal fue que, aunque los juicios de importancia de los lectoreśs deficientes no estaban altamente correlacionados con los de los adultos, mostraban una consistencia en lo que ellos consideraban importante. Los datos que apoyan esta conclusión provienen de las correlaciones entre los índices de importancia de cada sujeto y los índices medios de importancia de los demás miembros de su grupo. Las correlaciones medias fueron: $0,337,0,370$ y 0,616 para los lectores deficientes, los buenos lectores y los adultos, respectivamente.

Estos datos argumentan fuertemente que los lectores deficientes del grado 8 no eran idiosincráticos en sus juicios de importancia. Dado que estos lectores tienen ideas consistentes acerca de lo que es importante en un texto, la pregunta sería ¿Qué tiempos de información consideran importantes los lectores deficientes?

Para contestar esto, y usando la medida de Chi cuadrado, se identificaron las frases que los lectores deficientes tendían a seleccionar como importantes y que no seleccionaban ni los adultos ni los buenos lectores. Este análisis reveló diferencias significativas $(\mathrm{p}<0,05)$ entre los grupos, para 65 de las 224 frases en los ocho textos (29 por 100). Estas 65 frases podian clasificarse en cuatro grupos: (a) 16 frases seleccionadas más por los lectores fluidos (buenos lectores y adultos) que por los deficientes; (b) 17 frases seleccionadas más por niños que por adultos; (c) 17 frases seleccionadas más por adultos que por niños y (d) 15 frases seleccionadas más por los lectores deficientes que por los lectores fluidos. Estas úl= timas 15 frases son las más pertinentes en este estudio.

Al examinar las frases elegidas con más frecuencia por los lectores deficientes es difícil identificar una caracteristica propia. Sin embargo, en este estudio, las frases nos dan una idea de lo que le interesa al lector deficiente. Consideremos por ejemplo, las frases seleccionadas por un número significativamente mayor de 
lectores deficientes en el texto titulado Las ciudades en el siglo XIX (ver Tabla 1). Parece que estas frases están llenas de detalles visuales ricos, detalles que tal vez captan el interés de los lectores. Por el contrario, los que leen con fluidez parecen definir más la importancia en relación con el texto.

Un tercer hallazgo importante fue que la relación entre la información que los lectores deficientes juzgan como importante y aquella que incluyen en los resúmenes, no fue muy grande. Esta relación fue mayor para los buenos lectores y mayor aún para los adultos. En el grupo total la correlación entre los índices de los lectores deficientes y la frecuencia con que incluian esa información en un resumen era de 0,186 . Los coeficientes correspondientes a los buenos lectores y a los adultos fue mucho mayor, $0,598 \mathrm{y}$ 0,750 respectivamente.

Así, mientras los lectores deficientes eran consistentes en cuanto a la importancia dada, con un nivel que se aproximaba al de los buenos lectores, este nivel de importancia juzgado, tenía menos influencia a la hora de determinar lo que incluian los lectores deficientes en sus resúmenes.

Los resultados anteriores plantean la pregunta de por qué la correlación entre lo considerado importante y lo incluido en el resumen era tan baja en estos lectores deficientes. Para responder a esta cuestión, se llevaron a cabo diversos análisis. El primero de ellos examinó la posibilidad de que los efectos de la posición serial tuviera una gran influencia sobre qué ideas se incluían en los resúmenes. Las frases de cada pasaje se dividieron en cuartiles según su posición serial, con un mismo número de frases aproximadamente en cada cuartil. Luego se determinaba para cada cuartil la proporción de ideas incluidas en los resúmenes. Los resultados de estos análisis se muestran en la figura 1 . Los adultos mostraron una curva característica que indicaba que un tercio de las ideas $(0,34)$ de los resúmenes procedía del primer cuartil. Luego las cantidades decrecian en el segundo $(0,23)$ y en el tercero

TABLA 1

Frases que mostraron patrones de selección de lo importante significativamente distintos para los factores fluidos y menos fluidos.

\section{Las ciudades en el siglo XIX}

* En los últimos años del siglo xix, las ciudades de los EE. UU. crecián más rápidamente de lo que se habria pensado. Pero, a medida que las ciudades crecian, surgían los problemas.

Uno de los problemas eran los suburbios, con edificios sucios y atestados de gentes a los que se les llamaban pisos. En los suburbios, las enfermedades se propagaban a gran velocidad cuando la gente se ponía enferma. En los suburbios muy poblados, la gente tiraba la basura por las ventanas, y se acumulaba en enormes montones en las calles $y$ en los callejones. Los insectos y las ratas de la basura aumentaban las enfermedades de estos barrios.

Con tanta gente como había en las ciudades, la basura de repente llegó a ser un problema fuera de los suburbios. Nadie adivinaba que las ciudades encontrarían algún modo de recogida de basura. Porque, incluso en Nueva York, la ciudad más grande del país, la basura siempre se la comían los cerdos por la calle.

Se levantaban nuevos edificios casi de un día para otro. Muchos estaban hechos pobremente y todos ellos apiñados. La mayoría estaban construidos, al menos en parte, de madera. El peligro de incendio aumentaba. Las ciudades comenzaron a padecer terribles fuegos que rápidamente hacían arder barrios enteros. Chicago tuvo uno de los peores incendios. Se destruyó la mayor parte de la ciudad y cientos de personas murieron - quedaron beridas. Si las ciudades tenian que ser seguras, los edificios deberían ser mejores y se necesitarian los cuerpos de bomberos.

* El crimen era otro de los problemas de la ciudad. Bueno, siempre ha habido criminales. Pero, al igual que otras personas, se sentían especialmente atraídos por la ciudad. La única diferencia era que los criminales iban a la ciudad por motivos distintos. Tal cantidad de gente y los múltiples negocios, ofrecian a los ladrones más objetivo y habia tanta población que era dificil capturar a los bandidos. $A$ veces una banda se hacia cargo de un barrio de la ciudad y basta la policia tenia miedo de entrar en él.

Casi todo el mundo se daba cuenta de que las ciudades necesitaban ayuda*. Pero mucha gente creía que los ayuntamientos no tenian por qué resolver los nuevos problemas surgidos, tales como los suburbios, la basura o el crimen.

Nota: Las frases subrayadas fueron seleccionadas como importantes $(p<0,05)$ por más lectores deficientes que buenos lectores o adultos. Las frases con asterisco las eligieron significativamente como más importantes $(\mathrm{p}<0,05)$ los buenos lectores y los adultos que los lectores deficientes. 
$(0,18)$, para volver a aumentar en el último cuartil $(0,23)$. Esta curva es casi iđéntica a la obtenida por Kintsch y Kosminsky (1977) en su trabajo con resúmenes realizados por alumnos de college. Los buenos lectores tendían a aproximarse al modelo producido por los adultos, pero con menos tendencia a incluir información de la parte final del texto. Las proporciones de los cuatro cuartiles en estos sujetos fueron 0,35 , $0,26,0,20$ y 0,17 respectivamente

Comparemos las curvas de los adultos y de los buenos lectores con la de los lectores deficientes. Estos, extrajeron, casi la mitad de las ideas del primer cuartil $(0,44)$, luego la proporción decrecía regularmente en el segundo $(0,25)$ y en el tecero $(0,16)$. Los lectores deficientes obtuvieron el 0,13 de su información del último cuartil mientras que los adultos obtuvieron 0,23 y los buenos lectores 0,17 .
Dados estos modelos de inclusión de la información en los resúmenes, sería instructivo considerar el análisis paralelo que examinaba los efectos de la posición serial de las ideas seleccionadas por los sujetos como las más importantes de los textos. Estos resultados se muestran en la figura 2. De nuevo notaremos la curva característica tanto de los adultos como de los buenos lectores. Las proporciones de los cuatro cuartiles en los adultos fueron $0,35,0,22,0,19$ y 0,22 . Para los buenos lectores las cifras fueron $0,29,0,22,0,21$ y 0,24 . Los lectores deficientes, sin embargo, seleccionaron aproximadamente una cuarta parte de las ideas de cada uno de los cuartiles

Cuando los datos de las figuras 1 y 2 fueron sometidos a un análisis de varianza de medidas repetidas 3 (grupo) por 4 (cuartil), se hallaron efectos significativos tanto para los datos incluidos en el resumen como para los datos de selec-

FIGURA 1

Proporción de frases de cada cuartil del texto incluidas en los resumenes.

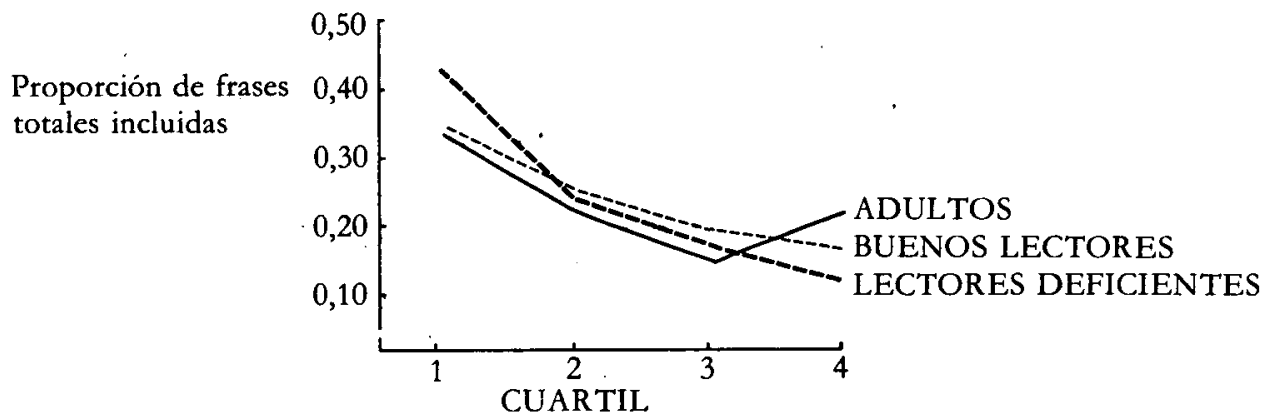

FIGURA 2

Proporción de frases de cada cuartil del texto que fueron seleccionadas como una de las cinco mas importantes.

Proporción de frases totales seleccionadas

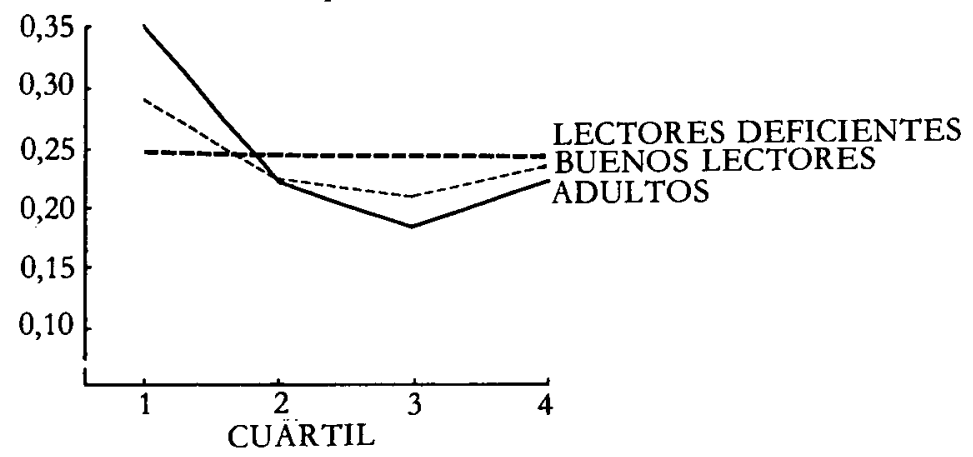


ción de lo importante. Los detalles de estos análisis y sus respectivas medidas post hoc se explican a continuación.

Ideas incluidas en los resúmenes. Cuando la variable dependiente fue la proporción de ideas incluidas en el resumen (figura 1), tanto el efecto del cuartil como los efectos del cuartil $\mathrm{X}$ grupo fueron significativos $(F(3,327)=84,59, \mathrm{p}<0,0001$ y $F(6,327)=6,81, p<0,0001$, respectivamente). Se midieron los efectos simples para describir más completamente la interacción grupo $\mathrm{X}$ cuartil. Estos análisis indicaron que había efectos significativos del cuartil dentro de cada grupo: lectores deficientes, $F(3,327)=62,53$, $\mathrm{p}<0,005$; buenos lectores, $\mathrm{F}(3,327)=$ $20,37, \mathrm{P}<0,005$; adultos, $\mathrm{F}(3,327)=$ $15,30, \mathrm{p}<0,005$. Las comparaciones Scheffé hechas dentro de cada uno de los tres grupos revelaron que tanto los lectores buenos como los deficientes incluían significativamente más ideas del primer cuartil que del segundo y más del segundo que de los otros dos. Los dos últimos cuartiles no difirieron significativamente entre sí. Los adultos también incluyeron significativamente más ideas del primer cuartil que del segundo y más del segundo que del tercero. Sin embargo, incluyeron más ideas del cuarto que del tercero. Posteriores análisis indicaron que el segundo y el cuarto cuartil no diferian significativamente entre sí.

Las medidas de los efectos principales simples se usaron también para examinar las diferencias entre los tres grupos dentro de cada cuartil. Estos análisis revelaron las diferencias significativas entre los grupos en los cuartiles primero y cuarto, $\mathrm{F}(2,436)=13,83, \mathrm{p}<0,005 \mathrm{y}$ $F(2,436)=10,76, p<0,005$ respectivamente. Las comparaciones Scheffé en el primer cuartil indicaron que los lectores deficientes diferian significativamente entre sí. Las mismas comparaciones en el cuartil cuarto indicaron que los adultos incluían significativamente más ideas que los buenos lectores y que éstos incluían más ideas que los deficientes.

Selección de lo importante. Cuando la variable dependiente del análisis de varianza de medidas repetidas 3 (grupo) por 4 (cuartil), fue la proporción de las selecciones de frases importantes (figura 2), fueron significativos los efectos del cuartil y de la interacción grupo $\mathrm{X}$ cuartil.
$(\mathrm{F}(3,327)=14,75, \mathrm{p}<0,001$ y $\mathrm{F}(6,237)$ $=3,97, \mathrm{p}<0,001$ respectivamente). Se llevaron a cabo unos análisis más minuciosos para examinar la interacción grupo $\mathrm{X}$ cuartil. Las medidas de los efectos simples dentro de cada grupo reveló que la posición del cuartil no tenía efectos significativos sobre qué ideas seleccionaban los lectores deficientes como importantes; las seleccionaron de forma igual de cada cuartil. Por el contrario, los efectos del cuartil fueron significativos tanto en los lectores buenos, F $(3,327)$ $=5,10, \mathrm{p}<0,005$ como en los adultos, $F(3,327)=18,64, p<0,005$. Las medidas post hoc revelaron que los buenos lectores seleccionaban significativamente más ideas del primer cuartil que del segundo y del tercero, pero no del cuarto. Además, los últimos tres cuartiles no diferían significativamente entre sí. Los adultos por el contrario, seleccionaron más ideas del primer cuartil que de cualquiera de los otros y los últimos tres cuartiles no diferían significativamente entre sí.

El último grupo de medidas de los efectos simples examinó las diferencias entre los tres grupos dentro de cada cuartil. Los tres difirieron significativamente en el primer cuartil $\mathrm{F}(2,436)=$ $13,20, \mathrm{p}<0,005$. Las comparaciones de Scheffé indicaron que los adultos seleccionaban más ideas del primer cuartil que los lectores deficientes. Los buenos lectores, aunque seleccionaban más ideas que los deficientes, no diferían significativamente ni de éstos ni de los adultos̀. También se encontraron diferencias significativas en el tercer cuartil, $F(2,436)$ $=3,48, \mathrm{p}<0,05$. Las medidas post hoc revelaron que los adultos seleccionaron menos ideas que los lectores deficientes. Los buenos lectores también eligieron menos ideas que los deficientes, pero la diferencia no fue significativa. No hubo tampoco diferencias significativas entre los grupos para los cuartiles segundo y cuarto.

Los resultados de estos análisis de medidas repetidas y las pruebas posteriores sugieren que los lectores deficientes están usando dos estrategias no relacionadas: una para decidir qué hay que incluir en el resumen y otra para seleccionar qué frases son las más importantes. Las pautas de los buenos lectores y de los 
adultos sugieren, sin embargo que usan la sensibilidad hacia lo importante como guía tanto para las tareas de inclusión como de selección.

Los análisis posteriores revelaron aún otro aspecto importante de la falta decoordinación entre lo que los lectores deficientes consideran importante y lo que incluye en sus resúmenes. Se halló el indice de importancia media de las ideas de cada cuartil del texto que se incluyeron en los resúmenes. Después se halló para cada cuartil, el indice de importancia media de cada sujeto para las ideas que no habian sido incluidas. Estos datos se muestran en la tabla 2. Los adul- tos y de una forma coherente, incluyeron en sus resúmenes la información que habian calificado de importante. Los buenos lectores hicieron también lo mismo y suprimieron aquella información que habian valorado como menos importante. Pero los lectores deficientes, por el contrario, eligieron la información que más habían valorado tan sólo en los tres primeros cuartiles. A medida que leen el texto, las diferentes medias entre el indice de las inclusiones y el de las supresiones se hace cada vez menor, hasta que en el último cuartil, la información incluida en los resúmenes tenia un índice de importancia media por debajo del dado a la información suprimida.

TABLA 2

Indices de importancia media de las frases incluidas en los resumenes $y$ de las frases suprimidas, por cuartil

\begin{tabular}{|c|c|c|c|c|c|c|c|}
\hline \multirow[b]{2}{*}{ Grupo } & & \multicolumn{6}{|c|}{ Cuartil del texto } \\
\hline & & 1 & 2 & & 3 & & 4 \\
\hline Adultos & $\begin{array}{l}\text { inclusiones } \\
\text { supresiones } \\
\text { diferencia }^{1}\end{array}$ & $\begin{array}{r}4.16(.37) \\
3.38(.36) \\
.79(.30)\end{array}$ & $\begin{array}{r}3.95(.44) \\
3.33(.38) \\
.63(.29)\end{array}$ & $\begin{array}{r}4.02 \\
3.25 \\
.77\end{array}$ & $\begin{array}{l}(.81) \\
(.43) \\
(.73)\end{array}$ & $\begin{array}{r}4.06 \\
3.34 \\
.72\end{array}$ & $\begin{array}{l}(.40) \\
(.42) \\
(.29)\end{array}$ \\
\hline Buenos lectores & $\begin{array}{l}\text { inclusiones } \\
\text { supresiones } \\
\text { diferencia } 1\end{array}$ & $\begin{array}{r}3.69(.56) \\
3.32(.49) \\
.37(.49)\end{array}$ & $\begin{array}{r}3.56(.56) \\
3.30(.46) \\
.26(.31)\end{array}$ & $\begin{array}{r}3.67 \\
3.23 \\
.44\end{array}$ & $\begin{array}{l}(.56) \\
(.47) \\
(.50)\end{array}$ & $\begin{array}{r}3.56 \\
3.36 \\
.20\end{array}$ & $\begin{array}{l}(.73) \\
(.45) \\
(.70)\end{array}$ \\
\hline Lectores deficientes & $\begin{array}{l}\text { inclusiones } \\
\text { supresiones } \\
\text { diferencia } 1\end{array}$ & $\begin{array}{r}3.34(.60) \\
3.14(.58) \\
.20(.37)\end{array}$ & $\begin{array}{r}3.35(.85) \\
3.19(.49) \\
.16(.84)\end{array}$ & $\begin{array}{r}3.38 \\
3.28 \\
.11\end{array}$ & $\begin{array}{r}(1.09) \\
(.49) \\
(.95)\end{array}$ & $\begin{array}{r}3.12 \\
3.30 \\
.18\end{array}$ & $\begin{array}{r}(1.34) \\
(.54) \\
(1.33)\end{array}$ \\
\hline
\end{tabular}

Nota: N." de casos: adultos $=37$; buenos lectores $=39$; lectores deficientes $=37$. Los números entre paréntesis indican la desviación típica.

1 Diferencias medias.

Estos resultados nos ofrecen más pruebas de que los lectores fluidos mantienen una fuerte relación entre lo que consideran importante y lo que incluyen en sus resúmenes. Sin embargo, los lectores deficientes muestran menos coherencia entre lo que incluyen en su resumen y lo que han calificado de importante. Además, los lectores deficientes eran más propensos a los efectos contrarios de la posición serial. Es decir, los lectores deficientes incluian menos información de la última parte del texto y lo que incluian era poco importante según sus propios juicios.

\section{E1 uso de las transformaciones del resumen}

El tercer grupo de análisis examinó la posibilidad de que los lectores deficien- tes fracasaran al usar o usaran ineficazmente aquellas transformaciones que utilizaban los buenos lectores.

Los análisis preliminares revelaron que los tres grupos no diferían en el número de palabras usadas en los resúmenes $F \cdot(2,109)=1,2, p<0,1$. Sin embargo, difirieron significativamente en la proporción de ideas que cada uno suprimió de los textos originales $F(2,109)=$ $10,39, \mathrm{p}<0,0005$. Las medidas post hoc revelaron que los lectores deficientes y los buenos lectores suprimían más ideas del texto original que los adultos. La proporción media de las ideas del texto que fueron suprimidas por los lectores deficientes, los buenos lectores y los adultos fue de $0,78,0,78$ y 0,72 respectivamente. Estos datos indican que los adultos tienen más aptitud para 
transmitir más ideas sin usar más palabras. Además el hecho de que los lectores deficientes y los buenos lectores no difirieran en el número de ideas omitidas hace más interesante la comparación de las ideas incluidas.

El resultado principal de este tercer conjunto de datos fue que había unas tendencias evolutivas claras en el uso de cada tipo de reglas. Advirtamos que la adquisición de la destreza lectora lleva a usar menos las repeticiones y las combinaciones enlazadas y a usar más las combinaciones y las invenciones. Este modelo se expresa en la figura 3 .

Esto se confirmó por procedimientos estadísticos. Un análisis de medidas repe- tidas 3 (grupo) por 4 (uso de la regla) produjo efectos significativos para el uso de la regla y para la interacción grupo $\mathrm{X}$ uso de la regla, $F(3,327)=60,99, p<$ 0,0001 y $F(6,327)=15,18, p<0,0001$, respectivamente. Las medidas más detalladas indicaron que los lectores deficientes utilizaban menos combinaciones que los buenos lectores, $F(2,436)=35,5$, $\mathrm{p}<0,005$. Esto es importante puesto que las combinaciones eran las transformaciones que más usaban los adultos. Además, aunque las diferencias no eran muy significativas, las tendencias evolutivas eran evidentes en el resto de las reglas. Aparentemente, los lectores deficientes fracasan al utilizar o usan ineficazmente aquellas reglas que utilizan los buenos lectores.

FIGURA 3

Proporción relativa del uso de las transformaciones

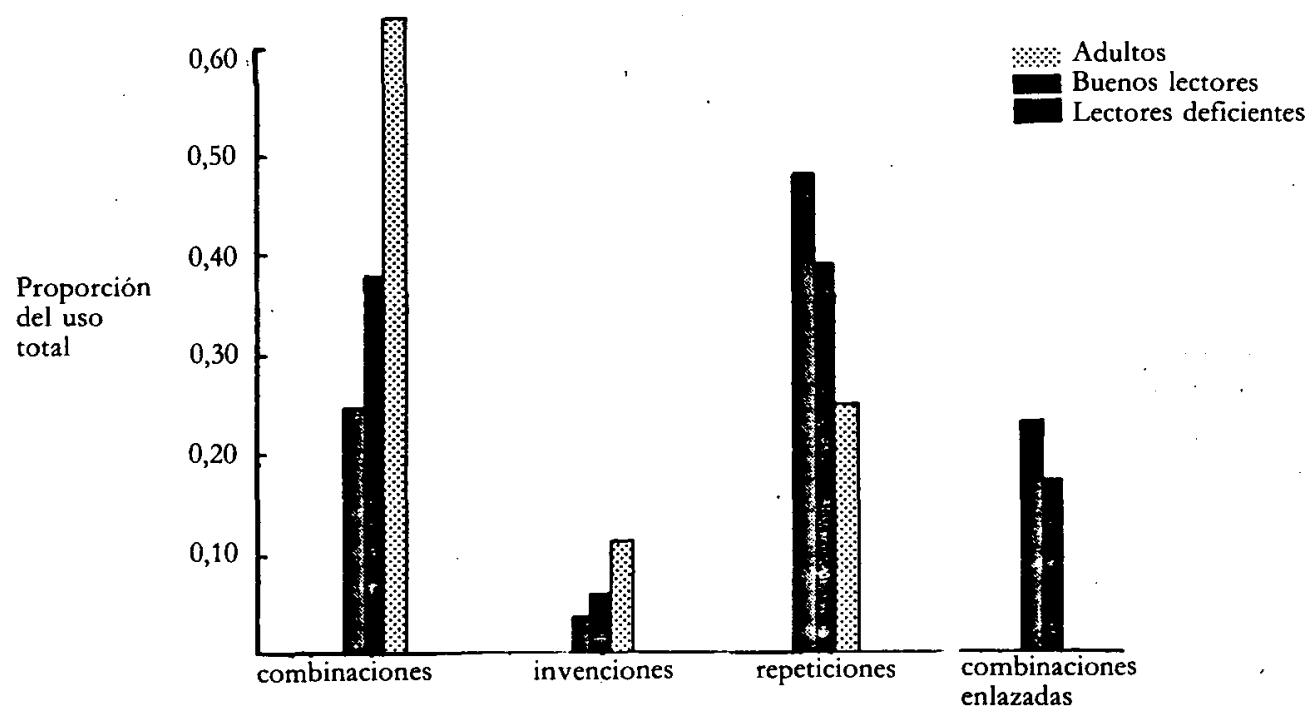

TRANSFORMACIONES

\section{La relación entre el uso de estrategia y la ejecución}

El último grupo de análisis estudió qué influencia tenian las diferencias en el uso de estrategia, sobre la aptitud para ejecutar la tarea de resumir y sobre la aptitud para comprender lo que se había leído. Se usó una regresión múltiple jerárquica (Cohen y Cohen, 1975) para construir y probar un modelo que usaba el resumen y las medidas de la ejecución en la comprensión como variables de- pendientes. Puesto que la regresión jerárquica supone que las variables entran en la ecuación de una manera teórica determinada, es necesario alguna explicación acerca del orden de las variables.

La primera variable que se introdujo en la ecuación fue la puntuación del CI en el IPAT Culture Fair. Esta medida de CI no verbal se incluyó para considerar la posibilidad de que las diferencias en la ejecución se debieran principalmente a diferencias en inteligencia. 
Las siguientes variables de la ecuación fueron la velocidad y la precisión en la decodificación. Estas dos medidas se obtuvieron en cada niño, cuando leía la lista de palabras en voz alta a uno de los experimentadores. La velocidad de decodificación era el tiempo en segundos que le llevaba al niño leer las 100 palabras. La precisión en la decodificación venía dada por el número de palabras falladas. I.a velocidad de decodificación y la precisión entraron como segunda y tercera variable, porque al menos en algunas teorias de la lectura, son un prerrequisito para la comprensión. Además, la aptitud para decodificar se cita a menudo como la fuente principal de las diferencias individuales en la comprensión. El hecho de que el $\mathrm{CI}$ y la aptitud para decodificar entraran antes que las variables de interés y justificaran la varianza tanto como fuera posible, proporcionaron una medida más conservadora de las hipótesis referentes a las relaciones entre el uso de la estrategia y la comprensión de orden superior.

Las variables cuarta y quinta del modelo fueron contrastes ortogonales basados en el nivel de respuesta dado a las preguntas de la entrevista que trataban de la conciencia de la tareá. El primer contraste comparó el nivel de respuesta 1 (una indicación clara de que el resumen incluye las ideas importantes) con el nivel de respuesta 2 (una indicación imprecisa) y el segundo contraste comparó los niveles de respuesta 1 y 2 con el nivel de respuesta 3 (no hay indicación).

La sexta variable que se introdujo en la ecuación fue una medida de cada niño de la sensibilidad hacia lo importante. Esta medida se obtuvo calculando la correlación entre los índices individuales de importancia y los índices medios de importancia de los adultos.

La séptima variable del modelo fue una medida del uso de la regla eficaz. Esta medida se obtuvo calculando la proporción de combinaciones e invenciones sobre el total de las transformaciones usadas por cada sujeto. Puesto que los adultos tienden a usar preferentemente estos dos tipos de transformaciones, se pensó que los niños que usaban una proporción alta de combinaciones e invenciones tenían un patrón del uso de las reglas más maduro.
El resto de las variables que formaban parte de la ecuación eran las interacciones de doble sentido entre cada uno de los efectos principales.

El orden de las variables que median la conciencia de la tarea, la sensibilidad hacia lo importante y el uso de las reglas estaba basado en el modelo de la comprensión presentado por Kintsch y van Dijk (1978). Ellos establecían que:

los propósitos del lector al leer, controlan la aplicación de los macro-operadores. La representación formal de estos propósitos es el esquema. Este determina qué microproposiciones o qué generalizaciones de microproposiciones son pertinentes y asi, qué partes del texto forman lo esencial.

Para los propósitos del modelo de regresión, se asumió que el lector fluido realiza la tarea con la conciencia de que resumir un texto es reducirlo a lo esencial. Luego, el sujeto identifica qué ideas deben incluirse y lógicamente aquellas que se desechan. Entonces, cuando el lector identifica los elementos relevantes y los no relevantes, se usan las macro-reglas para reducir el texto a un resumen. Este modelo se usó para examinar la ejecución de los niños al hacer resúmenes, al contestar a las preguntas de elección múltiple relacionadas con los textos experimentales y en el subtest de comprensión lectora del Stanford Achievement Test.

Calidad del resumen. La primera ecuación de regresión examinó la relación entre las diferencias de estrategia y la calidad de los resúmenes de los niños. La medida seleccionada para determinar la calidad de cada resumen de los niños, fue una puntuación obtenida calculando la correlación biserial puntual entre qué frases originales ha incluido el niño (1) o excluido (0) en el resumen y la proporción de adultos que también incluyeron esas frases en sus resúmenes. Así, esos niños que incluyeron las frases que también habian introducido una gran proporción de adultos, tenían correlaciones que se aproximaban a 1. A la inversa, aquellos niños cuyos resúmenes se basaban en frases que no habian sido utilizadas por muchos adultos tenían correlaciones que tendian a 0 .

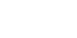

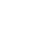


Los resultados de la ecuación de regresión jerárquica que tiene como variable dependiente esta correlación, se muestran en la tabla 3. El CI del IPAT Culture Fair explicaba una proporción de varianza significativa, el 16,94 por 100. Ni las medias de decodificación ni ninguno de los contrastes que implicaban el nivel de conciencia de la tarea fueron significativos. La sensibilidad hacia lo importante explicaba una proporción considerable de varianza, el 16,4 por 100. La proporción del uso de la regla eficaz también justificó una parte significativa de la varianza, el 4,10 por 100 ninguna de las interacciones fue significativa.

Comprensión del texto. La segunda ecuación de regresión examinó la relación entre las diferencias de estrategia y la comprensión de los textos experimentales. Estos datos se muestran en la tabla 4. La variable dependiente es el número medio de preguntas de elección múltiple que los niños contestaron correctamente.
El CI del IPAT Culture Fair explicó una proporción significativa de varianza, el 9,36 por 100 . La precisión en la decodificación también justificó parte de la varianza, el 4,98 por 100 . Ninguno de los contrastes que implicaban el nivel de conciencia de la tarea alcanzó significación. Sin embargo, la siguiente variable, la sensibilidad hacia lo importante, explicó el 5,31 por 100 de la varianza. El uso de la regla eficaz justificó un 3,55 por 100 de la varianza, una proporción que se aproxima a la significación.

Hubo dos interacciones significativas, el CI X la precisión en la decodificación que explicó el 6,27 por 100 y la interacción velocidad de decodificación $\mathrm{X}$ contraste 2 que justificó el 5,80 por 100 de la varianza. Un estudio de estas dos interacciones indicó que probablemente eran resultado del efecto-techo puesto que la mayoria de los niños contestaron bien las preguntas de elección múltiple. Este fue el caso de la interacción CI del IPAT $\mathrm{X}$ precisión en la decodificación. Sin em-

TABLA 3

Proporción de varianza de las puntuaciones en los resúmenes.y pruebas de significación

\begin{tabular}{lcc}
\hline \multicolumn{1}{c}{ Vatiable } & F & $\begin{array}{c}\text { Porcentale } \\
\text { de la } \\
\text { varianza }\end{array}$ \\
\hline CI del IPAT Culture Fair & $17,29 * *$ & 16,94 \\
Velocidad de decodificación & $<1$ & 0,05 \\
Precisión en la decodificación & 1,50 & 1,47 \\
Contraste 1 & 1,66 & 1,62 \\
Contraste 2 & $<1$ & 0,04 \\
Sensibilidad hacia lo importante & $16,73 * *$ & 16,40 \\
proporción del uso de la regla eficaz & $4,23 *$ & 4,15 \\
IPAT X velocidad de decodificación & $<1$ & 0,08 \\
IPAT X precisión en la decodificación & $<1$ & 0,04 \\
IPAT X contraste 1 & 1,20 & 1,18 \\
IPAT X contraste 2 & $<1$ & 0,02 \\
IPAT X sensibilidad hacia lo importante & $<1$ & - \\
IPAT X proporción del uso de la regla eficaz & $<1$ & 0,20 \\
velocidad de decodificación X precisión & $<1$ & 0,19 \\
velocidad de decodificación X contraste 1 & $<1$ & 0,02 \\
velocidad de decodificación X contraste 2 & 1,48 & 1,45 \\
velocidad de decodificación X sensibilidad hacia lo importante & $<1$ & 0,79 \\
velocidad X proporción del uso de la regla eficaz & $<1$ & - \\
precisión en la decodificación X contraste 1 & $<1$ & 0,85 \\
precisión X contraste 2 & $<1$ & 0,63 \\
precisión X sensibilidad hacia lo importante & $<1$ & 0,94 \\
precisión X proporción del uso de la regla eficaz & $<1$ & 0,97 \\
contraste 1 X sensibilidad hacia lo importante & $<1$ & 0,36 \\
contraste 1 X proporción del uso de la regla eficaz & $<1$ & $-1,16$ \\
contraste 2 X sensibilidad hacia lo importante & 3,23 & 3,16 \\
contraste 2 X proporción del uso de la regla eficaz & 2,33 & 2,28 \\
sensibilidad hacia lo importante X uso de la regla eficaz & 1,14 & 1,09 \\
\hline
\end{tabular}

Nota: $\mathrm{N}<74, \mathrm{r}^{2}=0,55$. Todas las variables independientes tienen 1 grado de libertad. 


\begin{tabular}{|c|c|c|}
\hline Variable & $\mathbf{F}$ & $\begin{array}{c}\text { Porcentaje } \\
\text { de la } \\
\text { varianza }\end{array}$ \\
\hline $\mathrm{Cl}$ del IPAT Culture Fair & $9,09 * *$ & 9,36 \\
\hline Velocidad de decodificación & $<1$ & 0,11 \\
\hline precisión en la decodificación & $4,83^{*}$ & 4,98 \\
\hline contraste 1 & $<1$ & 0,01 \\
\hline contraste 2 & 2,23 & 2,29 \\
\hline sensibilidad hacia lo importante & $5,16 *$ & 5,31 \\
\hline proporción del uso de la regla eficaz & 3,44 & 3,55 \\
\hline IPAT X velocidad de decodificación & $<1$ & 0,46 \\
\hline IPAT $X$ precisión en la decodificación & $6,09 *$ & 6,27 \\
\hline IPAT $X$ contraste 1 & $<1$ & 0,05 \\
\hline IPAT X contraste 2 & $<1$ & 0,35 \\
\hline IPAT X sensibilidad hacia lo importante & $<1$ & 0,13 \\
\hline IPAT X proporción del uso de la regla eficaz & $<1$ & - \\
\hline velocidad de decodificación X precisión & 2,65 & 2,73 \\
\hline velocidad de decodificación X contraste 1 & 2,79 & 2,87 \\
\hline velocidad de decodificación $\mathrm{X}$ contraste 2 & $5,64 *$ & 5,80 \\
\hline velocidad X sensibilidad hacia lo importante & 1,53 & 1,57 \\
\hline velocidad X proporción del uso de la regla eficaz & $<1$ & - \\
\hline precisión en la decodificación $\mathrm{X}$ contraste 1 & 3,83 & 3,94 \\
\hline precisión $X$ contraste 2 & $<1$ & - \\
\hline precision X sensibilidad hacia lo importante & $<1$ & 0,01 \\
\hline precisión X proporción del uso de la regla eficaz & $<1$ & 0,76 \\
\hline contraste $1 \mathrm{X}$ sensibilidad hacia lo importante & $<1$ & 0,28 \\
\hline contraste 1 X proporción del uso de la regla eficaz & $<1$ & 0,27 \\
\hline contraste $2 \mathrm{X}$ sensibilidad hacia lo importante & $<1$ & 0,93 \\
\hline contraste $2 \mathrm{X}$ proporción del uso de la regla eficaz & $<1$ & 0,27 \\
\hline sensibilidad hacia lo importante $X$ uso de la regla eficaz & $<1$ & 0,33 \\
\hline
\end{tabular}

Nota: $\mathbf{N}=74, \mathrm{r}^{2}=0,53$. Todas las variables independientes tienen 1 grado de libertad.

$* \mathrm{p}<0,05$.

$* * \mathrm{p}<0,005$.

bargo, la interacción entre el contraste 2 y la velocidad de decodificación produjo un resultado interesante. Un examen de los datos reveló un sujeto que estaba casi dos desviaciones típicas por encima de la media de la velocidad de decodificación del grupo (más lento) y cuyas respuestas a las preguntas de la entrevista indicaron que el sujeto creía que resumir un texto era memorizarlo. Aparentemente, este punto de vista del sujeto sobre la tarea experimental y su lenta velocidad de decodificación habían interactuado hasta tal punto que responder a las preguntas de comprensión al final del texto fue muy difícil. Su puntuación media de comprensión fue 2 sobre 5 cuando el promedio de todos los sujetos fue 4,02.

Stanford Achievement Test. La última ecuación de regresión estudió la relación entre las diferencias de estrategia y una variable dependiente que no tenía restricción en una escala, la puntuación de los niños en el subtest de comprensión lectora del Stanford Achievement Test. Estos resultados se expresan en la tabla
5. El CI del IPAT Culture Fair explicó una gran proporción de varianza, el 20,08 por 100 . Al contrario que los resultados anteriores de los análisis de regresión, la velocidad de decodificación y no la precisión, justificó una proporción de varianza significativa, el 8,71 por 100 . Ninguno de los contrastes que implicaban un nivel de conciencia de la tarea se aproximaba a la significación. Sin embargo, la sensibilidad hacia lo importante explicó el 5,4 por 100 de la varianza en las puntuaciones de lectura en los niños, proporción que fue casi la misma para esta variable en el análisis de regresión que tenía a la comprensión del texto como variable dependiente. Ni el uso de la regla eficaz ni ninguna interacción fue significativa.

Al considerar las tres ecuaciones de regresión, el resultado más sorprendente es que la aptitud para identificar los elementos importantes del texto explica una proporción significativa de la varianza en las tres medidas dependientes, incluso después de que se hayan tenido en cuen- 


\begin{tabular}{lcc}
\hline \multicolumn{1}{c}{ Variable } & F & $\begin{array}{c}\text { Porcentaje } \\
\text { de la } \\
\text { varianza }\end{array}$ \\
\hline CI del IPAT Culture Fair & $21,01 * * *$ & 20,08 \\
Velocidad de decodificación & $9,11 * *$ & 8,71 \\
precisión en la decodificación & 3,71 & 3,55 \\
contraste 1 & $<1$ & 0,26 \\
contraste 2 & 2,23 & 2,13 \\
sensibilidad hacia lo importante & $5,65 *$ & 5,40 \\
proporción del uso de la regla eficaz & 1,83 & 1,75 \\
IPAT X velocidad de decodificación & 1,93 & 1,84 \\
IPAT X precisión en la decodificación & $<1$ & - \\
IPAT X contraste 1 & 1,69 & 1,62 \\
IPAT X contraste 2 & $<1$ & 0,67 \\
IPAT X sensibilidad hacia lo importante & $<1$ & 0,64 \\
IPAT X proporción del uso de la regla eficaz & 1,60 & 1,53 \\
velocidad de decodificación X precisión & $<1$ & 0,19 \\
velocidad de decodificación X contraste 1 & $<1$ & 0,09 \\
velocidad de decodificación X contraste 2 & $<1$ & 2,13 \\
velocidad X sensibilidad hacia lo importante & 2,23 & 0,14 \\
velocidad X proporción del uso de la regla eficaz & $<1$ & 0,04 \\
precisión en la decodificación X contraste 1 & $<1$ & 0,13 \\
precisión X contraste 2 & $<1$ & 0,87 \\
precisión X sensibilidad hacia lo importante & $<1$ & 1,84 \\
precisión X proporción del uso de la regla eficaz & 1,92 & . \\
contraste 1 X sensibilidad hacia lo importante & $<1$ & 0,31 \\
contraste 1 X proporción del uso de la regla eficaz & $<1$ & 0,08 \\
contraste 2 X sensibilidad hacia lo importante & $<1$ & 0,09 \\
contraste 2 X proporción del uso de la regla eficaz & $<1$ & 0,82 \\
sensibilidad hacia lo importante X proporción del uso de la regla & 1,17 & 1,12 \\
\hline
\end{tabular}

Nota: $\mathrm{N}=74, \mathbf{r}^{2}=0,56$. Todas las variables independientes tienen 1 grado de libertad.

$* \mathrm{p}<0,05$.

$* * \mathrm{p}<0,005$.

$* * * \mathrm{P}<0,0005$.

ta el CI y la aptitud para decodificar. Este es un argumento convincente de que las dificultades de orden superior en la comprensión, pueden estar ligadas a los déficit de estrategia. También indica que la aptitud para identificar los elementos importantes de un texto, es una destreza de estrategia que subyace tanto a la comprensión como al resumen.

Segundo, el uso de la regla eficaz parece ser una destreza de estrategia más especifica de la tarea. Es decir, la aptitud para sintetizar un texto en un resumen mediante el uso de las transformaciones identificadas en este estudio, no se relacionaba significativamente con la aptitud para comprender ese texto. Esta conclusión se basa en el hecho de que el uso de la regla eficaz sólo justifica una proporción significativa de varianza, en la regresión en que la variable dependiente fue la puntuación del resumen.

Tercero, para la mayoría, la conciencia de la tarea no era un problema significativo $\mathrm{ni}$ al resumir ni al comprender lo que se había leído. Esto no es sorprendente dado que la mayoría de los niños revelaron que eran conscientes de que un aspecto de la tarea de resumir era incluir la idea principal. El hecho de que el factor conciencia de la tarea no fuera significativo, también enfatiza la importancia de las medidas de conexión basadas en datos introspectivos para las medidas de ejecución (Ryan, 1981). De otro modo, no habría sido difícil asumir que las diferencias en la claridad de las respuestas de los niños, hubiera sido un indicador de la existencia de diferencias de estrategia subyacentes.

\section{Resumen y Conclusiones}

En conjunto, los datos no mantienen la primera hipótesis de que algunos de los problemas de los lectores deficientes en los resúmenes, deriven de la confu- 
sión sobre las demandas de la tarea. Los resultados indican que la mayoría de los estudiantes del grado 8 de este estudio, sabian que un resumen debe incluir las ideas importantes de un texto. Los datos también indican que el nivel de claridad en las definiciones de "resumir" estaba poco relacionado, si es que lo estaba algo, con la ejecución de comprender o resumir un texto. Sin embargo, merece la pena repetir que una medida más sensible que las preguntas escritas, podría haber arrojado unas conclusiones diferentes.

Los datos confirman la segunda hipótesis de que algunos lectores deficientes tengan dificultad al identificar la información que los adultos consideran importante. Los buenos lectores están más de acuerdo con los adultos que los lectores deficientes, respecto a las concepciones sobre lo importante. Sin embargo, los lectores deficientes eran tan coherentes en sus juicios de importancia como los buenos lectores. Así, el problema, no era que los lectores deficientes se comportaran de una manera idiosincrática o que les faltara sensibilidad hacia lo importante, sino que tenian puntos de vista diferentes sobre qué ideas de un texto eran las importantes.

Según estos resultados, podemos especular que los lectores fluidos podían identificar la importancia basándose en los juicios de restricción textual y contextual. En otras palabras, aunque hubiesen encontrado algunos- elementos importantes del texto por sus intereses o por sus antecedentes, también habrian podido señalar lo que el autor consideraba importante mediante el uso de las señales contextuales. Por el contrario, los lectores menos fluidos basaban las selecciones de la información importante probablemente sólo por restricciones contextuales. Los tipos de información que eligieron como importantes parecian ser aquellos que les interesaban y no la información que el autor había establecido como más importante del texto. Para estudios futuros, sería necesario precisar esta suposición usando un análisis de la estructura del texto basado en la teoría, para identificar más específicamente los factores que influyen en la elección que hacen los buenos lectores y los deficientes.
Otra diferencia sobresaliente entre los buenos lectores y los deficientes es el grado de relación entre lo que los sujetos consideran importante y lo que incluyen en sus resúmenes. Una explicación de la baja correlación que se da en los lectores deficientes, es que los lectores fluidos incluían las ideas según lo que percibian en el texto, mientras que las ideas seleccionadas por los lectores deficientes estaban afectadas por la posición serial. Para la investigación futura, seria importante saber por qué la posición serial tiene un efecto tan negativo $y$ saber cómo enseñar a los niños a superarlo.

Otro resultado importante que concierne a la sensibilidad hacia lo importante, es que explicaba proporciones de varianza significativas en las puntuaciones del resumen y en las medidas de la aptitud para la comprensión incluso cuando ya se habían considerado las diferencias en el CI y en la aptitud para decodificar. Estos resultados nos proporcionan la evidencia de que las dificultades de orden superior pueden estar unidas a los déficit de estrategia. Estos datos también indican que la aptitud para identificar los elementos importantes del texto, es una destreza de estrategia que subyace tanto al resumen como a la comprensión.

Consideremos por qué la sensibilidad hacia lo importante es tan vital. Cuando leen, los-lectores fluidos, pueden usar los signos textuales y el conocimiento anterior para identificar los elementos importantes del texto. Estos elementos se usan luego para construir una representación interna del mensaje del autor (Kintsch y van Dijk, 1978). La suposición antes hecha indicaba que los lectores deficientes tendrian dificultad en usar los signos textuales. Sin tal guía, seria muy dificil construir una representación precisa $y$ organizada de lo que el autor quiere comunicar. Además, dada la importancia de la organización en la memoria, la falta de tal representación haría que la información inicialmente se recordara menos, que entretanto se olvidara más probablemente y que se recuperara con menos probabilidad cuando fuese necesario (Meyer, 1977).

Los datos de este estudio también confirman la tercera hipótesis de que los

\section{.}

(n)


lectores deficientes fracasan al utilizar o utilizan ineficazmente, aquellas transformaciones del resumen usadas por los buenos lectores. Estos resultados replican y amplian los de Day (1980), de Marshall y Glock (1978-79) y de Tierney y Bridge (1979), que indicaban que los lectores deficientes tenían dificultad en integrar las proposiciones individuales en unidades más grandes. Además, el hecho de que el uso de la regla eficaz explique una parte significativa de la varianza en las puntuaciones del resumen, pero no en las de la comprensión, indica que esta destreza de estrategia es más relevante en la tarea de resumir que en el proceso global de la comprensión.

El hecho de que el uso de la regla eficaz esté significativamente relacionado con las puntuaciones del resumen, pero no con las puntuaciones de la comprensión, puede ayudar a clarificar la relación entre el resumen y la comprensión. Una explicación sería que la tarea de resumir no sólo requiere que el lector construya una representación interna del mensaje del autor, representación que también es necesaria para la comprensión, sino que hay que tomar otras decisiones secundarias acerca de la importancia relativa de los elementos en esa representación interna (Brown y Day, 1980; Johnson, 1978). Por otra parte, parece que estas operaciones secundarias requieren el control activo del lector en mayor grado que el proceso de la comprensión, que resulta inicialmente de la representación interna. Los lectores deficientes tropiezan con dificultades en ambos estadios de la tarea. No sólo tienen problemas al construir una representación interna del mensaje del autor, sino que también los tienen cuando se necesitan las operaciones secundarias para hacer un resumen.

Podemos esbozar algunas consecuencias pedagógicas a partir de este estudio. La primera proviene del hecho de que la sensibilidad hacia lo importante, explica una proporción significativa de la varianza de las puntuaciones de la comprensión de los niños. Los maestros pueden determinar la sensibilidad hacia lo importante que tienen los niños, cuando existe un indicio de dificultades en la comprensión. Los métodos para determinarla, pueden ser formales e informales. cuál es la idea más importante del texto y por qué, podremos obtener una información diagnóstica útil. Un método más formal implicaria unos procedimientos similares a los usados en este trabajo, en donde los niños podrían identificar las frases más importantes de un texto cuidadosamente elegido. Las elecciones del niño podrían compararse con las normas de lectores fluidos o con los resultados obtenidos en un análisis teórico de la estructura del texto. En cualquier caso, los maestros pueden hallar que los lectores deficientes necesitan un entrenamiento explícito en las estrategias de orden superior 'de la comprensión, además de, o en lugar de, un entrenamiento en las destrezas para la decodificación. Es importante resaltar este punto puesto que, hasta hace poco, ha sido siempre más fácil centrarse en los problemas de la decodificación, ya que se conocía lo que había que enseñar sobre la destreza para decodificar. Esto no quiere decir que una decodificación fluida no sea importante; realmente lo es. Sin embargo, puede ser necesario enseñar algunas /destrezas adicionales, particularmente aquellas que tienen que ver con la comprensión de grandes unidades de texto. Los resultados presentados en este estudio indican que la aptitud para identificar los elementos más importantes de un pasaje, puede incluirse en una de esas destrezas. Una cuestión importante para la investigación futura es encontrar algunas estrategias de enseñanza eficaces para alcanzar este objetivo.

Una segunda consecuencia, estrechamente relacionada con la primera, proviene del hallazgo de que los lectores deficientes muestran cierta consistencia en los tipos de frases que consideran importantes. Esto implica que, puesto que las frases que los lectores deficientes tendían a seleccionar como importantes, diferían de aquéllas seleccionadas por los adultos y los buenos lectores, las selecciones no tienen necesariamente carácter idiosincrático. Más bien parece que estos lectores se basan en unos criterios distintos de los usados por los lectores fluidos: Puede ser necesario llevar a los lectores deficientes a desarrollar criterios distintos y posiblemente más discriminatorios para decidir lo que es importante en un texto. Los maestros pueden facilitar mejor esta transición mostrando una mayor sensibi- 
lidad al hecho de que lo que parece ser una selección incorrecta desde la perspectiva del lector fluido, puede ser en realidad una elección bastante razonable según la perspectiva del lector menos fluido o del lector joven.

La tercera consecuencia se basa en los datos que indican que la tarea de resumir implica algunas estrategias además de las que son necesarias en la comprensión. Por otra parte, cuando los niños tienen problemas al resumir lo que han leído, los profesores.no deberian asumir automáticamente que los niños tienen dificultades a la hora de entenderlo. Aunque los problemas al resumir pueden ser sintomáticos de los problemas en la comprensión, tener dificultades al hacer un resumen no se limita necesariamente a problemas de comprensión. Parece ser que las dificultades de algunos niños al resumir, residen en las operaciones secundarias usadas para condensar y transformar un texto a lo esencial. Así, el entrenamiento de estos estudiantes en un intento por mejorar las aptitudes para la comprensión, puede no mejorar la ejecución de los requisitos de la tarea de resumir.

En conclusión, este estudio proporciona información que debería ser útil para determinar algunas de las fuentes de los problemas de orden superior en la comprensión que padecen muchos lectores deficientes. Los resultados reseñados aquí ayudan también a enfatizar el concepto de que existan otras dificultades en la comprensión lectora además de la existencia de destrezas inadecuadas para la decodificación, y que hacer un resumen va más allá de la comprensión de un texto.

\section{Referencias}

BAklik, L., y Brow', A. L.: Metacognitive skills in reading (Technical Rep. No. 188). Urbana: Universidad de Illinois, Center for the Study of Reading (ERIC Document Reproduction Service No. ED 195 932), 1980 .

Bi:RGi:R, N. S., y Plikfi:tTI, C. A.: «Reading skill and memory for spoken and written discourse». Journal of Reading Behavior, 1977, 9, 7-16.

Blatock, H. M., JR.: Social statistics. Nueva York: McGraw:Hill, 1979.

Brown, A. L., y DAY, J. D.: Strategies for summariqing texts: The development of expertise. Manuscrito inédito. Universidad de Illinois, 1980.

Brown, A. L., y SmILEY, S. S.: «Rating the importance of structural units of prose passages: A problem of metacognitive development”. Cbild Development, 1977, 48, 1-8.

CANNFY, G., y WiNOGRAD. P.: Scbemata for reading and reading comprehension performance (Tech. Rep. No. 120). Urbana: Universidad de Illinois, Center for the Study of Reading (ERIC Document Reproduction Service No. ED 169521 ), 1979.

CATTFLL, R. B., y CATTF.LL, A. K. S.: Handbook for the culture fair intelligence test. Champaign, IL: Institute for Personality and Ability Testing, 1960.

CLAY, M. M.: "Reading errors and self-correction behaviour. British Journal of Educational Psychology", $1969,39,47-56$.

COHEN, J., y COHF, P.: Applied multiple regression/correclation analysis for the bebavioral sciences. Hillsdale, N. J.: Erlbaum, 1975.

Collins, A., y Haviland, S. E.: Children's reading problems (Reading Education Rep. No. 8). Urbana: Universidad de Illinois, Center for the Study of Reading (ERIC Document Reproduction Service No. ED 172 188), 1979.

DAY, J. D.: Teaching summarization skills: $A$ comparison of training methods. Tesis doctoral inédita. Urbana: Universidad de Illinois, 1980

Denny, T., y WeINTRAuB, S.: «Exploring first grader's concepts of reading». The Reading Teacher, 1963, $16,363-365$.

Downing, J.: Reading and reasoning. Nueva York: Springer-Verlag, 1979.

DUNN, B. R., MATHEws, S. R., II, y BIEGER, G.: Individual differences in the recall of lower-level textual information (Tech. Rep. No. 150). Urbana: Universidad de Illinois, Center for the Study of Reading. (ERIC Document Reproduction Service No. ED 181 448).

EAMON, D. B.: «Selection and recall of topical information in prose by better and poorer readers». Reading Research Quarterly, 1978, 14, 244-257.

Education Development Chinter. Man: $A$ course of study. Washington, DC: Curriculum Development Association, 1970.

EHRI, L. C.: «Linguistic insight threshold of reading acquisition». En T. G. Waller \& G. E. MacKinnon (Eds.), Reading research: Advances in theory and practice (Pp. 63-114). Nueva York: Academic Press, 1979.

FRY, E.: «Fry's readability graph: Clarifications, validity, and extension to level 17». Journal of Reading, $1977,21,242-252$.

GinN BASIC RF.ADER. My do and learn book. Boston, MA: Ginn and Company, 1967.

GolinkOFF, R.: «A comparison of reading comprehension processes in good and poor comprehenders». Reading Research Quarterly, 1975-76, 11, 623-659. 
Holt DatABank SySTEM. Inquiring about American bistory. Nueva York: Holt, Rinehart \& Winston, $1976 \mathrm{a}$.

HOLt DATABANK SYSTHM. Inquiring about Cultures. Nueva York: Holt, Rinehart \& Winston, $1976 \mathrm{~b}$.

JoHNS, J. L.: «First grader's concepts about print». Reading Research Quarterly, 1980, 15, 529-549.

JOHNSON, N. S.: A structural analysis of the development of story recall and summarization. Tesis doctoral inedita, Universidad de California, San Diego, CA, 1978.

JOHNSTON, P.: Implications of basic research for the assessment of reading comprebension (Tech. Rep. No. 206). Urbana: Universidad de Illinois, Center for the study of Reading (ERIC Document Reproduction Service No. ED 201 987), 1981.

KFNNEDY, E. C.: Classroom approaches to remedial reading. Itaca, IL: Peacock Publishers, 1971.

KINTSCH, W., y KOZMINSKY, E.: «Summarizing stories after reading and listening». Journal of Educational Psycbology, 1977, 69, 491-499.

KINTSCH, W., y VAN DIjK, T. A.: "Toward a model of discourse comprehension and production". Psychological Review, 1978, 85, 363-394.

KIRK, R. E.: Experimental design: Procedures for the behavioral sciences. Belmont, CA: Wadsworth Publishing Company, 1968.

MARShaLl, N., y GloCk, M. D.: "Comprehension of connected discourse : A study of into the relationship between the structure of text and information recalled". Reading Research Quarterly, 1978-79, 14, 10-56.

MFYER, B. J. F.: "The structure of prose: Effects on learning and memory and implications for educational practice». En R. C. Anderson, R. J. Spiro and W. E. Montague (Eds.), Schooling and the acquisition of knowledge (pp. 179-200). Hillsdale, N. J.: Erlbaum, 1977.

MEYiR, B. J. F.; BRANDT, D. M., y BLUTH, G. J.: "Use of top-level structure in text: Key for reading comprehension of ninth-grade students). Reading Research Quarterly, 1980, 16, 72-103.

MEYtRS, M., y PARIS, S. G.: “Children's metacognitive knowledge about reading". Journal of Educational Psychology, 1978, 70, 680-690.

Noble: and Noble Basal Soclal Studiks Shriks. Many Americans-One Nation. Nueva York: Noble \& Noble Publishers, 1974.

PERFETTl, C. A., y LFSGOLD, A. M.: «Discourse comprehension and sources of individual differences». En M. A. Just \& P. A. Carpenter (Eds.), Cognitive processes in comprehension (pp. 141-183). Hillsdale, N. J.: Erlbaum, 1977.

PICHeRT, J. W.: Sensitivity to what is important in prose (Tech. Rep. No. 149). Urbana: Universidad de Illinois. Center for the Study of Reading (ERIC Document Reproduction Service No. ED 179 946), 1979.

RFID, J. F.: «Learning to think about reading». Educational Research, 1966, 9, 56-62.

RYAN, E. B.: «Identifying and remediating failures in reading comprehension: Toward an instructional approach for poor comprehenders». En T. G. Waller \& G.E. MacKinnon (Eds.), Advances in reading research, Nueva York: Academic Press, 1981, Vol. 2 223-261.

Smiley, S. S.; OAklfy, D. D.; Worthen, D., CAmpione, J. C., y Brown, A. L.: «Recall of thematically relevant material by adolescent good and poor readers as a function of written versus oral presenta- tion». Journal of Educational Psycbology, 1977, 69, 381-387.

TIERNEY, R. J., y BRIDGE, C. A.: "The functions of inferences: An extended examination of discourse comprehension". En M. L. Kamil \& A. J. Moe (Eds.) Twenty-Eighth Yearbook of the National Reading Conference (pp. 129-131). Clemson, SC: National Reading Conference, 1979.

VAN DIJK, T. A.: "Semantic macro-structures and knowledge frames in discourse comprehension». En M. A. Just, \& P. A. Carpenter (Eds.), Cognitive presses in comprebension (pp. 3-32). Hillsdale, N. J.: Erlbaum, 1977.

VAN DiJK, T. A.: "Relevance assignment in discourse comprehension». Discourse Processes, 1979, 2, 113. 126.

Webster's Third New International Dictionary of the Englishi Language. Springfield, MA: Merriam, 1964.

Winograd, P.: An examination of strategic differences in summarizing texts. Tesis doctoral inedita. Urbana: Universidad de Illinois, 1982.

WRIGHT, R. H.: Curious wys of common birds. Nueva York: Lothrop, Lee \& Shepard, 1971.

\section{Apéndice}

\section{Texto Original}

\section{Las Nutrias}

Hay dos clases de nutrias, las marinas y las de río. Las nutrias marinas viven casi todo el tiempo en agua salada. Las nutrias de río viven cerca de los riachuelos de agua dulce y en los ríos de interior.

Las nutrias marinas tienen una capa suntuosa de piel suave y gorda. El pelo es casi todo marrón con algunas pintas blancas en la cabeza y en el cuello. Es muy valioso. Las nutrias marinas han sido cazadas por su piel durante tanto tiempo que quedan muy pocos ejemplares vivos hoy en dia.

Las nutrias de mar tienen un cuerpo largo con patas cortas. Sus grandes pies tienen membranas interdigitales y forma de aleta. Sus rabos son gordos y planos. La forma de la cola y de los pies les ayuda a nadar. 
Las nutrias marinas obtienen toda la comida del mar. Se sumergen hasta una profundidad de 90 metros para conseguir su manjar favorito, los crustáceos. Comen boca arriba y usan el vientre como mesa.

En algunas partes del mar hay enormes lechos de algas flotantes. Las nutrias marinas descansan y duermen en estas camas. Enrollan las algas al cuerpo para no ser arrastradas por el agua. Duermen sobre la espalda y las madres sujetan a sus crias entre los brazos.

Las nutrias de rio se parecen mucho a las marinas pero son más pequeñas y no pesan tanto. Sus pies también tienen membranas y son unas nadadoras muy rápidas. Tienen una piel marrón que parece negra cuando está mojada.

Las nutrias de río comen peces, ranas, reptiles y otros animales que pescan en los ríos y lagos cercanos a sus madrigueras.

Las nutrias de río no viven todo el tiempo en el agua. Viajan muchas milias por tierra de un río a otro.

La nutria de río es uno de los pocos animales salvajes que juegan mucho cuando es adulta. Se deslizan sobre sus estómagos por las orillas de los ríos. Doblan las patas delanteras debajo de su cuerpo y se impulsan con las posteriores. Algunos de sus toboganes son tan empinados que las nutrias deben trepar a la parte de arriba dando un rodeo. 